\title{
29. DETAILS OF MAGNETIC-POLARITY TRANSITIONS RECORDED IN SEDIMENT CORES FROM DEEP SEA DRILLING PROJECT SITE 445, PHILIPPINE SEA
}

\author{
Hajimu Kinoshita, Department of Geophysics, Faculty of Science, Chiba University, Yayoi-cho, Chiba, Japan
}

\begin{abstract}
Measurement of natural remanent magnetization (NRM) of a $15-\mathrm{m}$ interval of nannofossil-chalk cores from DSDP Site 445 revealed the presence of four magnetic-polarity transitions at around $25 \mathrm{Ma}$. Detailed measurements of NRM variation could be made because of the recovery of unbroken cores.

The NRM was found to be stable with respect to alternating-field demagnetization. Magnetism studies on selected specimens indicated the presence of unoxidized titanomagnetite.

Two of the four polarity transitions were rapid, and the polarity changed within 6000 years. The other two transitions were slow, and the polarity switched over 25,000 to 30,000 years; these transitions have the following features in common with transition zones observed by several investigators: (1) intermediate directions of magnetization, (2) a drop in the intensity and NRM/susceptibility ratio, and (3) a drop in magnetization intensity coincident with the directional fluctuations. Saturation-magnetization and bulk-susceptibility measurements on selected samples show that the decrease in NRM intensity associated with polarity change is likely not due to low concentration of ferromagnetic minerals.

The present results support the model of field reversal in which the main dipole field decays to a low value and then builds up in the opposite direction. The present data show also that the geomagnetic dipole moment of reversed polarity is less stable than that of normal polarity.
\end{abstract}

\section{INTRODUCTION}

Studies on the geomagnetic-field reversals have been carried out by a number of investigators (see references). There is no need to emphasize that study of field reversals is important to explanation of the origin of the magnetic field of the earth.

It is well established that deep-sea-sediment cores present a continuous record of the directional fluctuations and polarity changes of the past geomagnetic field. However, the absolute value of the geomagnetic-field intensity is hardly obtainable from the sedimentary cores. This problem usually is overcome by measuring either the Koenigsberger ratio of the natural remanent magnetization (NRM/[initial susceptibility]) or the ratio of NRM to saturation remanent magnetization. Both ideas are based upon the fact that the intensity of remanent magnetization is proportional to the total amount of ferromagnetic minerals and to the intensity of the geomagnetic field at the moment of sedimentation.

During DSDP Leg 58, 892 meters of sediments was penetrated at Site 445 , in the southeastern part of the Daito Ridge $\left(25^{\circ} 31.36^{\prime} \mathrm{N}, 133^{\circ} 12.49^{\prime} \mathrm{E}\right)$. Below 140 meters sub-bottom, the sedimentary cores consisted mainly of consolidated nannofossil chalks and greenish mudstones. Between 417 and 445 meters sub-bottom, milky-colored, faintly laminated, undisturbed nannofossil chalk cores were recovered with a small number of disordering fractures within a 9-meter-long core. From preliminary NRM measurements, the cores were found to be magnetically stable and applicable to paleomagnetism studies.

\section{MAGNETIC MINERALS}

A concentrate of ferromagnetic minerals was obtained from each of several portions of the cores by means of a hand magnet. The separated magnetic fractions consisted of dark and a few lighter-colored fine grains. High-field-strength (3000 oe) measurements of $J_{s}$ as a function of temperature were made in a vacuum of $10^{-4} \mathrm{~mm} \mathrm{Hg}$; X-ray-diffraction and fluorescence analyses were performed on the magnetic separates to identify the minerals.

For $J_{s}$ measurements, the samples were heated and cooled gradually at a rate of 4 to $5 \mathrm{deg} / \mathrm{min}$. Examples of $J_{s}$ curves are shown in Figure 1. It is obvious that no drastic change in the ferromagnetic characteristics of the minerals occurred during heating. Curie temperatures are determinable from these curves.

$\mathrm{X}$-ray-diffraction shows lattice parameters of the ferromagnetic minerals of cubic symmetry. In addition, $\mathrm{X}$-ray fluorescence shows that the metallic component 

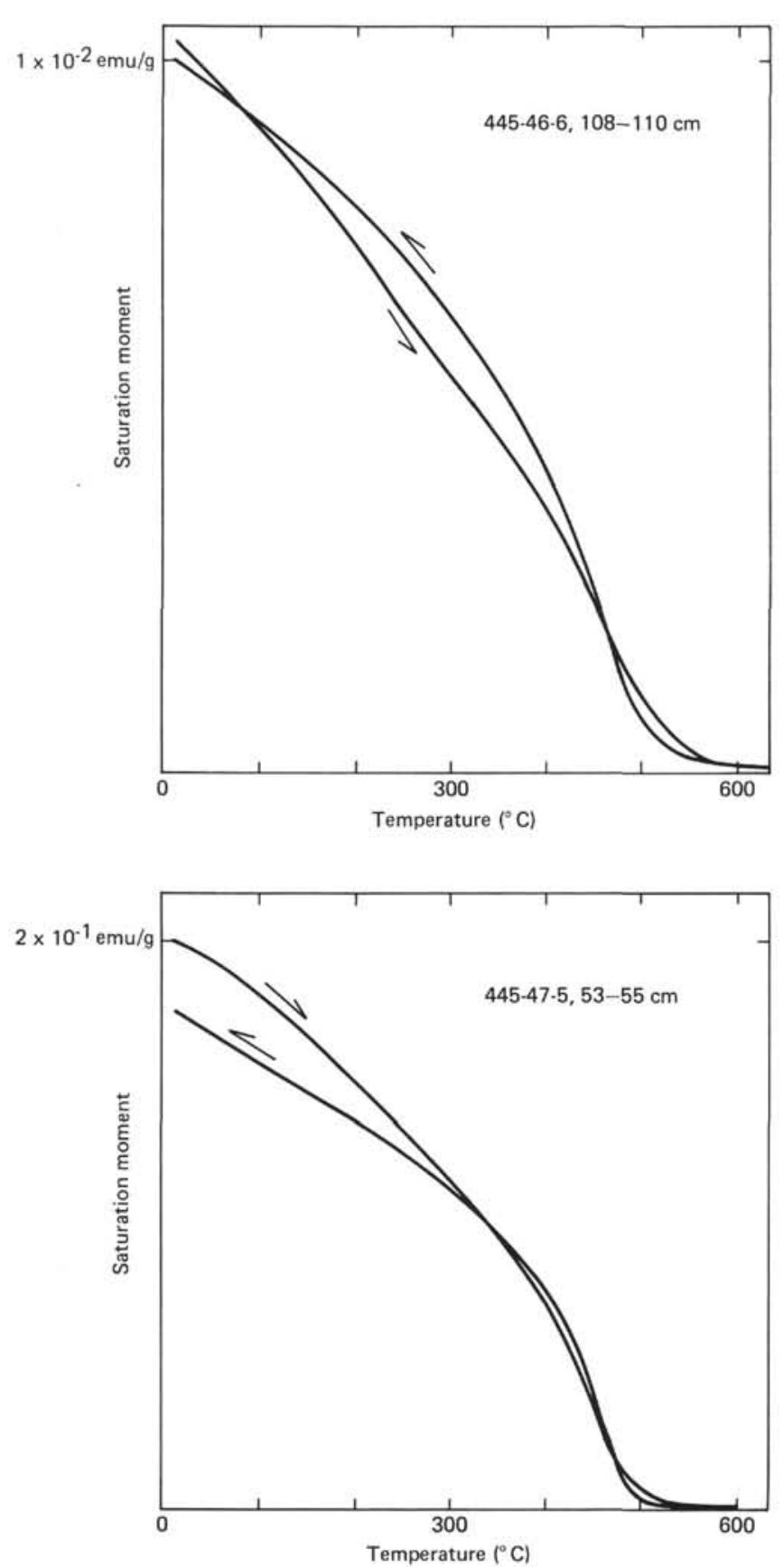

Figure 1. Two examples of $\mathrm{J}_{s}$ curves of ferromagnetic concentrates from pulverized minicores.

of the ferromagnetic minerals is mainly iron and titanium.

The results lead us to the conclusion that the main phase of the ferromagnetic minerals is titanomagnetite, with a low titanium content according to the diagram of Readman and O'Reilly (1972). Supposing that the ferromagnetic components are in stoichiometry, titanium contents are evaluated as part of a magnetite-ulvospinel solid solution: $x \mathrm{Fe}_{2} \mathrm{TiO}_{4} \cdot(1-x) \mathrm{Fe}_{3} \mathrm{O}_{4}$ (Table 1).
TABLE 1

Chemical Composition of Ulvospinel-Magnetite Solid Solution of Ferromagnetic Concentrates of Cores from Site 445

\begin{tabular}{lcccc}
\hline Sample & $\begin{array}{c}445-46-12, \\
2-4 \mathrm{~cm}\end{array}$ & $\begin{array}{c}445-46-6, \\
21-23 \mathrm{~cm}\end{array}$ & $\begin{array}{c}445-46-6, \\
108-110 \mathrm{~cm}\end{array}$ & $\begin{array}{c}445-47-5, \\
53-55 \mathrm{~cm}\end{array}$ \\
\hline$x$ values* & $0.1-0.4$ & $0.1-0.4$ & $0.05-0.07$ & $0.05-0.1$ \\
\hline
\end{tabular}

*Value $x$ corresponds to $x \mathrm{Fe}_{2} \mathrm{TiO}_{4} \cdot(1-x) \mathrm{Fe}_{3} \mathrm{O}_{4}$. Scatter of $x$ values is due to inconsistency between X-ray-diffraction and Curie-temperature data.

\section{AGE AND SEDIMENTATION RATE}

Ages of sediment cores were determined by paleontological studies on nannofossils, radiolarians and foraminifers. Plots of absolute ages for Cores 46 and 47 from Site 445 are shown in Figure 2. It is obvious that there should not be a hiatus in sedimentation processes around 24 to $25 \mathrm{~m}$.y. It is safe to assume therefore that the sedimentation rate for these sections was fairly constant when averaged over a time of 10,000 to 100,000 years. Fluctuation of sedimentation rate over times shorter than $1 \mathrm{~m} . \mathrm{y}$. cannot be resolved by existing techniques.

For the present study, about 150 minicores were cut from a 15-meter interval of the cores. The spacing of successive minicores was kept as close as possible to 10 $\mathrm{cm}$. Therefore, assuming a constant sedimentation rate $(15 \mathrm{~m} / \mathrm{m} . \mathrm{y}$.$) , each minicore of 25-\mathrm{mm}$ diameter and $25-\mathrm{mm}$ height would include a record of the past geo-

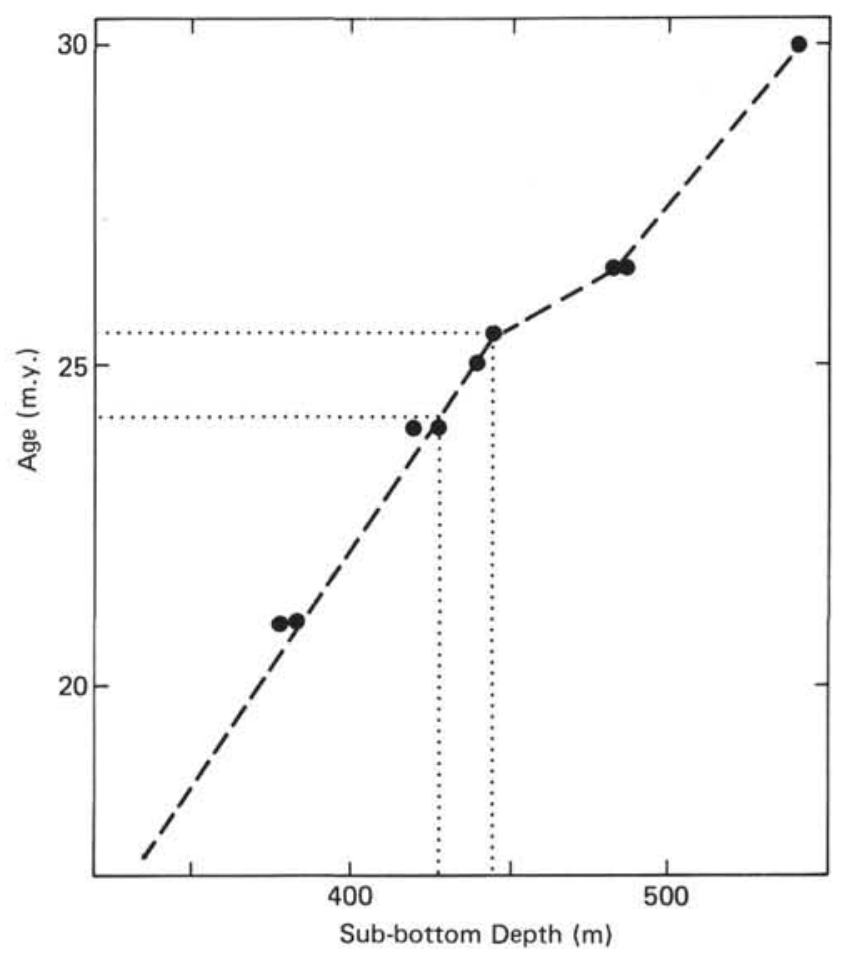

Figure 2. Paleontological age versus sub-bottom depth of cores. 
magnetic field smoothed out over a period of 1,650 years, with an average sampling rate of every 6,600 years. Supposing that the present migration rate of the geomagnetic non-dipolar field has been persistent, the period of 1,650 years is probably long enough to smooth out the wobble of the geomagnetic field.

\section{PALEOMAGNETIC RECORD}

Paleomagnetism measurements on minicores were carried out by standard techniques. The NRM was measured by a slowly spinning magnetometer connected to a computerized data-processing system. All specimens were demagnetized in an alternating field (AFD) of at least 150 oe peak field strength (Figure 3).

As noted earlier, Cores 46 and 47 were so undisturbed mechanically that we were able to obtain not only the inclination but the relative declination values for NRM and AFD. Where a fracture or disordering was found in the core, a discontinuity in the declination of NRM showed up. A correction of the declination is applied in a conventional way for convenience: (1) if the inclination of NRM differs little on opposite sides of a fracture, then the declination is supposed to be equal on opposite sides; (2) if the inclination differs by about $180^{\circ}$, then the declination is supposed to be different by $180^{\circ}$.
Results are shown in Figure 4, where AFD stands for demagnetization in an alternating field of 150 oe peak field strength. Declination values are only arbitrary (floating scale) and partly rearranged.

Details of field reversal are shown in Figure 5 for three different portions each $100 \mathrm{~cm}$ long. All paleomagnetism values are listed in Table 2.

\section{CONCLUSION}

Cores 46 and 47 consist mainly of nannofossil chalk deposited on a comparatively shallow ocean bottom. They were formed between 24 and $25.5 \mathrm{Ma}$, and the depositional rate was fairly constant, as indicated by paleontological age determination. Carriers of NRM are mainly titanomagnetite with a low titanium content, resembling the ferromagnetic components of andesitic or rhyolitic rocks.

If a constant depositional rate is assumed between 24 and $25.5 \mathrm{Ma}$, the time constant of each field reversal observed here is estimated to be less than 6,000 years, about 25,000 years, and 30,000 years (left to right in Figure 5, respectively). It is also evident that fluctuation of the direction of the regional magnetic field and the change in its field intensity were not identical for each field reversal. This phenomenon is reasonable if nondipolar field components are predominant at the time of field reversal, and if there is decay of the main dipolar

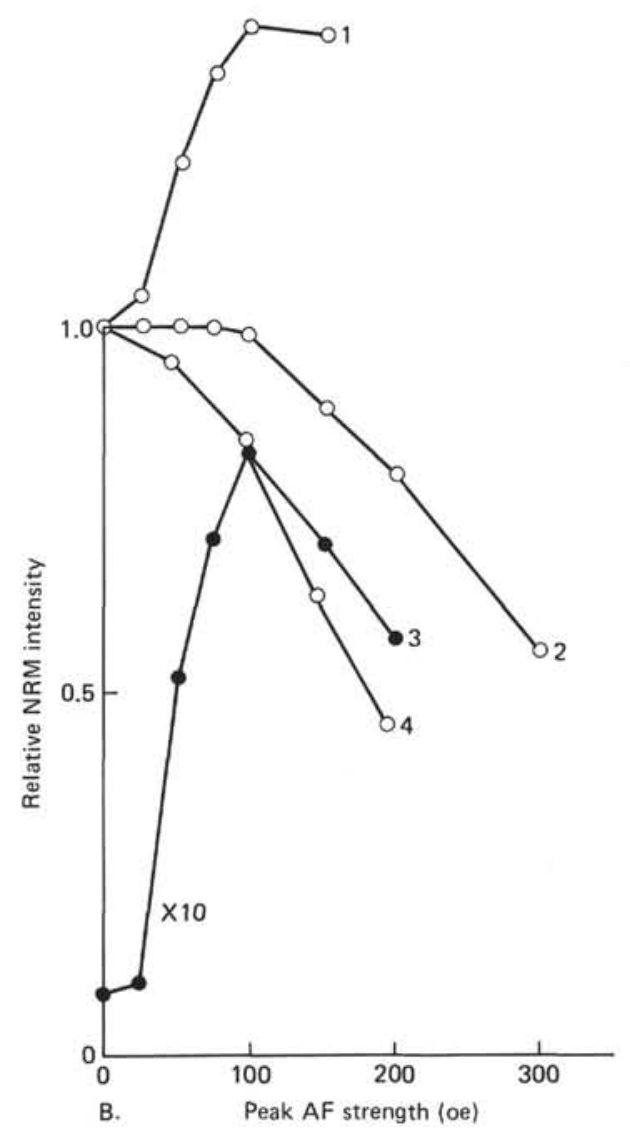

Figure 3. AFD data for nannofossil chalk. A. Normal polarity. B. Reversed polarity. 

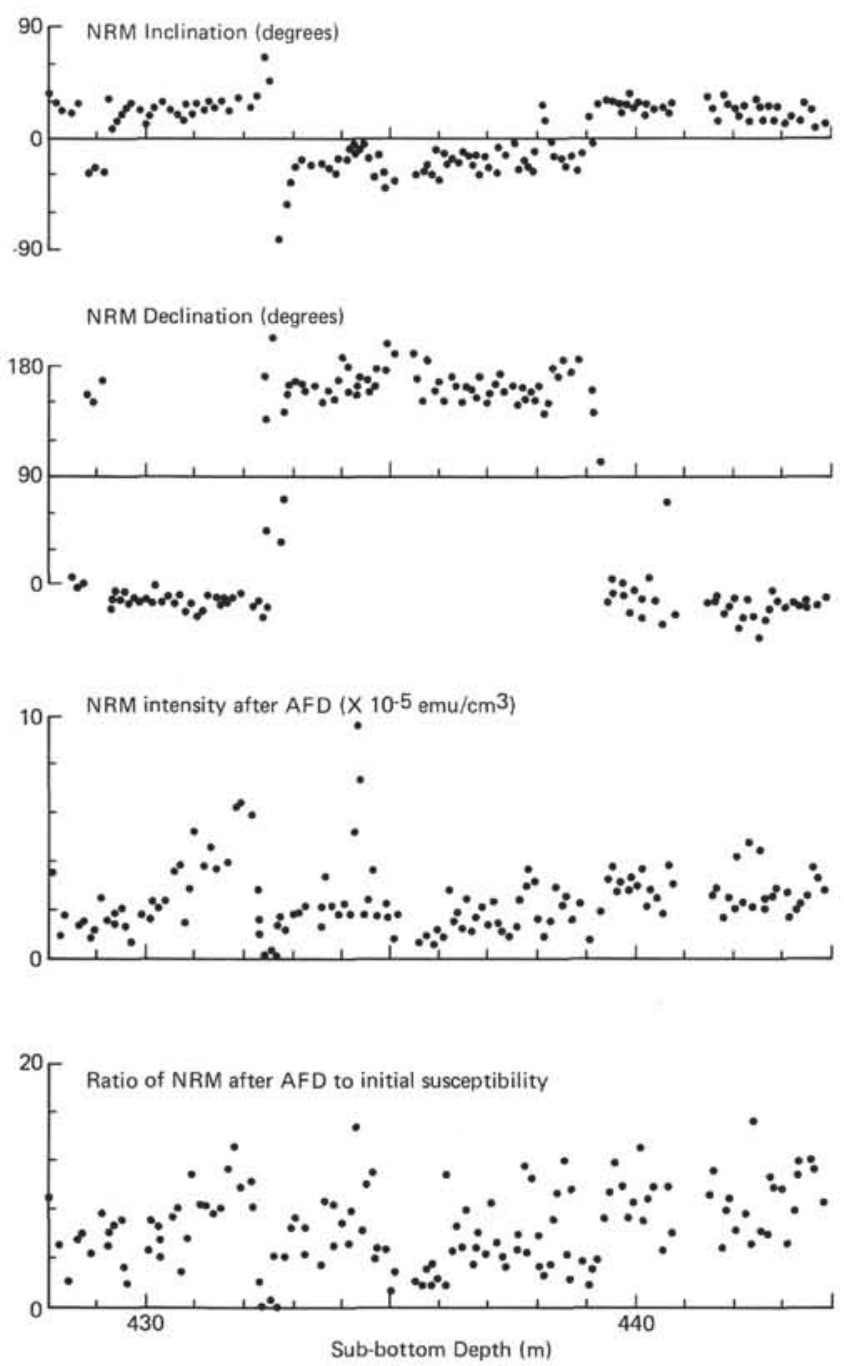

Figure 4. Paleomagnetism data plotted against subbottom depth.

field (Cox, 1968; Larson et al., 1971). However, observation shows that the dipole moment of the earth's magnetic field passed through the same path in two successive field reversals (Bucha, 1970). In such a case, the main dipole moment does not seem to have decayed when it was switching polarity.

It is obvious that the normal polarity lasted much longer than the reversed polarity around $24 \mathrm{Ma}$, and that the inclination of the normal polarity is much more stable than that of the reversed polarity. This feature supports the statistical argument presented by Wilson et al. (1972) and Dagley et al. (1974).

\section{ACKNOWLEDGMENTS}

The author would like to acknowledge Prof. H. Okada, Yamagata University, for his valuable discussions and unpublished data on the age of the nannofossil-chalk cores of DSDP Site 445. The author's thanks are due to Dr. Y. Furuta, University of Tokyo, for his great help in magnetism measurements and mineral analyses of the magnetic samples. The manuscript was critically read by Prof. K. Kobayashi and Prof. S. Uyeda, University of Tokyo.
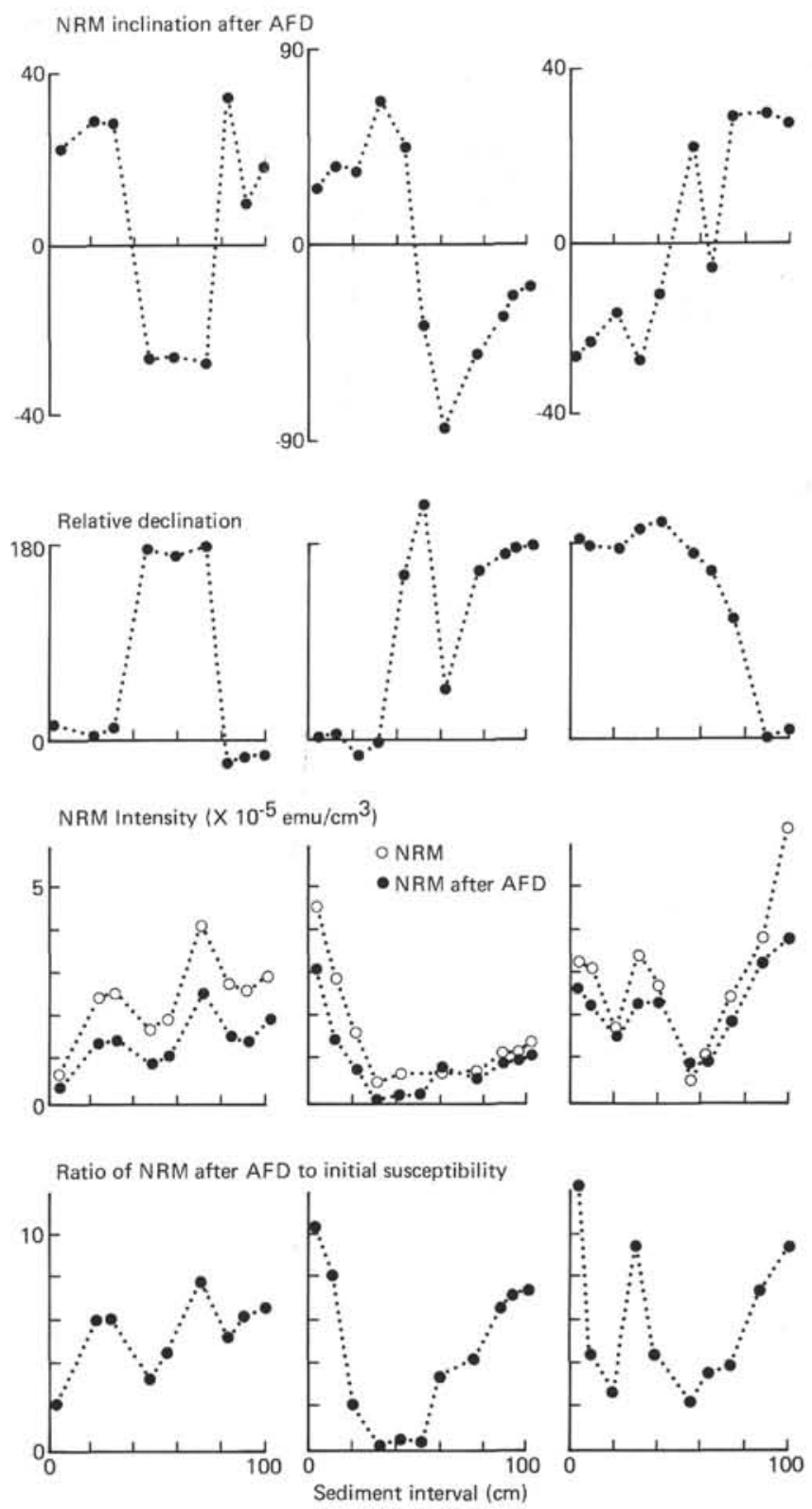

Figure 5. A details of paleomagnetism around the three field reversals shown in Figure 4.

\section{REFERENCES}

Bucha, V., 1970. Geomagnetic reversals in Quaternary revealed from a paleomagnetic investigation of sedimentary rocks. J. Geomagnet. Geoelec., 22, 253-272.

Cox, A., 1968. Length of geomagnetic polarity intervals. $J$. Geophys. Res., 73, 3247-3260.

Dagley, P. and Lawley, E., 1974. Paleomagnetic evidence for the transitional behaviour of the geomagnetic field. Geophys. J. Royal Astron. Soc., 36, 577-598.

Dunn, J. R., Fuller, M., Ito, H., and Schmidt, V. A., 1971. Paleomagnetic study of a reversal of the earth's magnetic field. Science, 172, 840-845.

Harrison, C. G. A., and Somayajulu, B. L. K., 1966. Behaviour of the Earth's magnetic field during a reversal. Nature, $212,1193-1195$. 
TABLE 2

Paleomagnetism Data for Sediment of Cores 445-46 and 445-47 (values before and after AFD)

\begin{tabular}{|c|c|c|c|c|c|c|c|}
\hline $\begin{array}{c}\text { Core } \\
\text { Interval } \\
\text { (cm) }\end{array}$ & $\begin{array}{l}\text { Peak } \\
\mathrm{AF} \\
\text { (oe) }\end{array}$ & $\begin{array}{c}J_{S} \\
\left(\times 10^{-5}\right. \\
\text { gauss) }\end{array}$ & $\begin{array}{l}\text { Suscepti- } \\
\text { bility } \\
\text { ( } \times 10^{-5} \\
\text { gauss/oe) }\end{array}$ & $\begin{array}{l}\text { Incli- } \\
\text { nation }\end{array}$ & $\begin{array}{c}\text { Declina- } \\
\text { tion } \\
\text { (relative } \\
\text { degrees) }\end{array}$ & $\begin{array}{l}\text { Rela- } \\
\text { tive } \\
\text { Inten- } \\
\text { sity }\end{array}$ & $\begin{array}{l}\text { Ratio of } \\
\text { NRM After } \\
\text { AFD to } \\
\text { Initial Sus- } \\
\text { ceptibility }\end{array}$ \\
\hline $\begin{array}{l}\text { Core 46, } \\
\text { Section } 2\end{array}$ & & & & & & & \\
\hline $1-3$ & 150 & $\begin{array}{l}5.17 \\
3.55\end{array}$ & $\begin{array}{c}0.39 \\
-\end{array}$ & $\begin{array}{l}36.8 \\
36.3\end{array}$ & $\begin{array}{l}233.9 \\
235.6\end{array}$ & $\overline{0.69}$ & $9 . \overline{17}$ \\
\hline $21-23$ & 150 & $\begin{array}{l}1.67 \\
1.00\end{array}$ & 0.20 & $\begin{array}{l}22.1 \\
26.0\end{array}$ & $\begin{array}{l}59.1 \\
63.2\end{array}$ & $\overline{0.60}$ & $5 . \overline{01}$ \\
\hline $28-30$ & 150 & $\begin{array}{l}2.85 \\
1.81\end{array}$ & $\stackrel{0.44}{-}$ & $\begin{array}{l}24.2 \\
23.6\end{array}$ & $\begin{array}{l}56.5 \\
60.5\end{array}$ & $\overline{0.64}$ & $\overline{4.11}$ \\
\hline $44-46$ & 150 & $\begin{array}{l}0.72 \\
0.41\end{array}$ & 0.20 & $\begin{array}{l}22.8 \\
21.6\end{array}$ & $\begin{array}{l}112.3 \\
118.6\end{array}$ & $\overline{0.57}$ & $\overline{2.05}$ \\
\hline $60-62$ & 150 & $\begin{array}{l}2.41 \\
1.44\end{array}$ & 0.24 & $\begin{array}{l}27.6 \\
28.0\end{array}$ & $\begin{array}{l}109.3 \\
109.1\end{array}$ & $\overline{0.59}$ & $5 . \overline{90}$ \\
\hline $69-71$ & 150 & $\begin{array}{l}2.52 \\
1.46\end{array}$ & $\begin{array}{c}0.24 \\
-\end{array}$ & $\begin{array}{l}27.9 \\
27.7\end{array}$ & $\begin{array}{l}115.5 \\
116.2\end{array}$ & $\overline{0.58}$ & $\overline{6.09}$ \\
\hline $86-88$ & 150 & $\begin{array}{l}1.70 \\
0.93\end{array}$ & $\begin{array}{c}0.29 \\
-\end{array}$ & $\begin{array}{l}-29.9 \\
-27.3\end{array}$ & $\begin{array}{l}-76.9 \\
-78.9\end{array}$ & $\overline{0.55}$ & $3 . \overline{25}$ \\
\hline $94-96$ & 150 & $\begin{array}{l}1.98 \\
1.13\end{array}$ & $\begin{array}{l}0.25 \\
-\end{array}$ & $\begin{array}{l}-28.4 \\
-26.8\end{array}$ & $\begin{array}{l}-79.7 \\
-83.1\end{array}$ & $0 . \overline{57}$ & $4 . \overline{51}$ \\
\hline $110-112$ & 150 & $\begin{array}{l}4.05 \\
2.59\end{array}$ & $\begin{array}{c}0.33 \\
-\end{array}$ & $\begin{array}{l}-29.1 \\
-27.5\end{array}$ & $\begin{array}{l}-74.9 \\
-73.1\end{array}$ & $\overline{0.64}$ & $\overline{7.87}$ \\
\hline $120-122$ & 150 & $\begin{array}{l}2.75 \\
1.58\end{array}$ & $\begin{array}{c}0.30 \\
-\end{array}$ & $\begin{array}{l}29.2 \\
33.2\end{array}$ & $\begin{array}{l}81.0 \\
85.6\end{array}$ & $\overline{0.59}$ & $5 . \overline{22}$ \\
\hline $129-131$ & 150 & $\begin{array}{l}2.51 \\
1.48\end{array}$ & 0.24 & $\begin{array}{r}15.1 \\
8.9\end{array}$ & $\begin{array}{l}82.5 \\
89.8\end{array}$ & $\overline{0.59}$ & $6 . \overline{17}$ \\
\hline $139-141$ & 150 & $\begin{array}{l}2.90 \\
1.92\end{array}$ & $\begin{array}{c}0.29 \\
-\end{array}$ & $\begin{array}{l}18.1 \\
17.8\end{array}$ & $\begin{array}{l}86.5 \\
91.4\end{array}$ & $\overline{0.66}$ & $\overline{6.60}$ \\
\hline $148-150$ & 150 & $\begin{array}{l}3.52 \\
2.06\end{array}$ & $\begin{array}{c}0.29 \\
-\end{array}$ & $\begin{array}{l}22.7 \\
21.7\end{array}$ & $\begin{array}{l}88.3 \\
89.0\end{array}$ & $\overline{0.59}$ & $7 . \overline{16}$ \\
\hline \multicolumn{8}{|l|}{ Section 3} \\
\hline $5-7$ & 150 & $\begin{array}{l}2.22 \\
1.26\end{array}$ & $\begin{array}{c}0.38 \\
-\end{array}$ & $\begin{array}{l}24.2 \\
24.1\end{array}$ & $\begin{array}{l}232.7 \\
238.3\end{array}$ & $\overline{0.57}$ & $3 . \overline{32}$ \\
\hline $14-16$ & 150 & $\begin{array}{l}1.45 \\
0.70\end{array}$ & $\stackrel{0.34}{-}$ & $\begin{array}{l}24.3 \\
28.3\end{array}$ & $\begin{array}{l}223.4 \\
226.3\end{array}$ & $\overline{0.48}$ & $\overline{2.04}$ \\
\hline $28-30$ & 150 & $\begin{array}{l}3.38 \\
2.32\end{array}$ & $\begin{array}{c}0.56 \\
-\end{array}$ & $\begin{array}{l}23.6 \\
23.6\end{array}$ & $\begin{array}{l}228.1 \\
228.1\end{array}$ & $\overline{0.69}$ & $\overline{4.14}$ \\
\hline $40-42$ & 150 & $\begin{array}{l}3.25 \\
1.83\end{array}$ & 0.29 & $\begin{array}{l}23.4 \\
23.4\end{array}$ & $\begin{array}{l}232.3 \\
235.4\end{array}$ & $0 . \overline{56}$ & $\overline{6.28}$ \\
\hline $50-52$ & 150 & $\begin{array}{l}2.26 \\
1.69\end{array}$ & $\begin{array}{l}0.35 \\
-\end{array}$ & $\begin{array}{l}13.3 \\
10.8\end{array}$ & $\begin{array}{l}229.4 \\
235.2\end{array}$ & $0 . \overline{75}$ & $\overline{4.84}$ \\
\hline $60-62$ & 150 & $\begin{array}{l}3.98 \\
2.57\end{array}$ & $\begin{array}{c}0.36 \\
-\end{array}$ & $\begin{array}{l}25.5 \\
20.2\end{array}$ & $\begin{array}{l}229.9 \\
228.0\end{array}$ & 0.65 & $\overline{7.19}$ \\
\hline $70-72$ & 150 & $\begin{array}{l}3.74 \\
2.23\end{array}$ & $\begin{array}{c}0.33 \\
-\end{array}$ & $\begin{array}{l}25.2 \\
25.6\end{array}$ & $\begin{array}{l}230.2 \\
241.3\end{array}$ & $\overline{0.60}$ & $\overline{6.80}$ \\
\hline $78-80$ & 150 & $\begin{array}{l}4.55 \\
2.39\end{array}$ & $\begin{array}{c}0.41 \\
-\end{array}$ & $\begin{array}{l}34.7 \\
27.3\end{array}$ & $\begin{array}{l}229.4 \\
233.4\end{array}$ & $\overline{0.53}$ & $\overline{5.88}$ \\
\hline $100-102$ & 150 & $\begin{array}{l}6.46 \\
3.72\end{array}$ & $\begin{array}{c}0.49 \\
-\end{array}$ & $\begin{array}{l}21.6 \\
22.8\end{array}$ & $\begin{array}{l}227.7 \\
233.8\end{array}$ & $\overline{0.58}$ & $\overline{7.64}$ \\
\hline $110-112$ & 150 & $\begin{array}{l}5.64 \\
3.89\end{array}$ & $\begin{array}{c}0.47 \\
-\end{array}$ & $\begin{array}{l}22.4 \\
21.1\end{array}$ & $\begin{array}{l}231.5 \\
227.1\end{array}$ & $\overline{0.69}$ & $8 . \overline{29}$ \\
\hline $122-124$ & 150 & $\begin{array}{l}2.77 \\
1.34\end{array}$ & $\begin{array}{c}0.46 \\
-\end{array}$ & $\begin{array}{l}19.0 \\
17.5\end{array}$ & $\begin{array}{l}226.6 \\
257.0\end{array}$ & $\overline{0.48}$ & $\overline{2.89}$ \\
\hline $132-134$ & 150 & $\begin{array}{l}5.37 \\
2.87\end{array}$ & $\begin{array}{c}0.49 \\
-\end{array}$ & $\begin{array}{l}22.1 \\
27.2\end{array}$ & $\begin{array}{l}232.8 \\
251.5\end{array}$ & $\overline{0.53}$ & $5 . \overline{80}$ \\
\hline $142-144$ & 150 & $\begin{array}{l}7.13 \\
5.34\end{array}$ & $\begin{array}{c}0.49 \\
-\end{array}$ & $\begin{array}{l}26.5 \\
21.1\end{array}$ & $\begin{array}{l}231.9 \\
233.7\end{array}$ & $0 . \overline{75}$ & $10 . \overline{91}$ \\
\hline \multicolumn{8}{|l|}{ Section 4} \\
\hline $1-3$ & 150 & $\begin{array}{l}8.41 \\
5.42\end{array}$ & $\underset{0.51}{-}$ & $\begin{array}{l}30.8 \\
28.9\end{array}$ & $\begin{array}{l}279.2 \\
293.5\end{array}$ & $\overline{0.64}$ & $10 . \overline{56}$ \\
\hline $15-17$ & 150 & $\begin{array}{l}5.97 \\
3.78\end{array}$ & $\begin{array}{c}0.44 \\
-\end{array}$ & $\begin{array}{l}26.2 \\
25.8\end{array}$ & $\begin{array}{l}297.0 \\
293.6\end{array}$ & $\overline{0.63}$ & 8.54 \\
\hline $28-30$ & 150 & $\begin{array}{l}6.52 \\
4.68\end{array}$ & $\begin{array}{c}0.57 \\
-\end{array}$ & $\begin{array}{l}30.0 \\
29.3\end{array}$ & $\begin{array}{l}295.7 \\
299.8\end{array}$ & $0 . \overline{72}$ & $\overline{8.20}$ \\
\hline $39-41$ & 150 & $\begin{array}{l}5.32 \\
3.77\end{array}$ & $\begin{array}{c}0.50 \\
-\end{array}$ & $\begin{array}{l}26.4 \\
25.3\end{array}$ & $\begin{array}{l}294.4 \\
297.5\end{array}$ & $0 . \overline{71}$ & $7 . \overline{54}$ \\
\hline $51-53$ & 150 & $\begin{array}{l}6.72 \\
4.58\end{array}$ & $\begin{array}{c}0.56 \\
-\end{array}$ & $\begin{array}{l}30.0 \\
27.1\end{array}$ & $\begin{array}{l}295.5 \\
299.3\end{array}$ & $\overline{0.68}$ & $8 . \overline{12}$ \\
\hline $59-61$ & 150 & $\begin{array}{l}6.08 \\
3.96\end{array}$ & $\begin{array}{c}0.40 \\
-\end{array}$ & $\begin{array}{l}27.1 \\
27.7\end{array}$ & $\begin{array}{l}291.3 \\
295.6\end{array}$ & $\overline{0.65}$ & 9.00 \\
\hline $69-71$ & 150 & $\begin{array}{l}6.60 \\
4.59\end{array}$ & $\begin{array}{c}0.40 \\
-\end{array}$ & $\begin{array}{l}25.2 \\
25.7\end{array}$ & $\begin{array}{l}291.1 \\
295.6\end{array}$ & $\overline{0.70}$ & $11 . \overline{50}$ \\
\hline $79-81$ & & 9.13 & 0.48 & 34.2 & 296.2 & - & - \\
\hline
\end{tabular}

TABLE 2 - Continued

\begin{tabular}{|c|c|c|c|c|c|c|c|}
\hline $\begin{array}{c}\text { Core } \\
\text { Interval } \\
\text { (cm) }\end{array}$ & $\begin{array}{l}\text { Peak } \\
\text { AF } \\
\text { (oe) }\end{array}$ & $\begin{array}{c}{ }_{S} \\
\left(\times 10^{-5}\right. \\
\text { gauss) }\end{array}$ & $\begin{array}{l}\text { Suscepti- } \\
\text { bility } \\
\left(\times 10^{-5}\right. \\
\text { gauss/oe) }\end{array}$ & $\begin{array}{l}\text { Incli- } \\
\text { nation }\end{array}$ & $\begin{array}{c}\text { Declina- } \\
\text { tion } \\
\text { (relative } \\
\text { degrees) }\end{array}$ & $\begin{array}{l}\text { Rela- } \\
\text { tive } \\
\text { Inten- } \\
\text { sity }\end{array}$ & $\begin{array}{l}\text { Ratio of } \\
\text { NRM After } \\
\text { AFD to } \\
\text { Initial Sus- } \\
\text { ceptibility }\end{array}$ \\
\hline & 150 & 6.35 & - & 33.7 & 299.5 & 0.70 & 13.20 \\
\hline $89-91$ & 150 & $\begin{array}{l}9.59 \\
6.48\end{array}$ & $\begin{array}{c}0.65 \\
-\end{array}$ & $\begin{array}{l}33.6 \\
33.5\end{array}$ & $\begin{array}{l}299.4 \\
301.1\end{array}$ & $\overline{0.67}$ & $9 . \overline{96}$ \\
\hline $111-113$ & 150 & $\begin{array}{l}8.88 \\
6.13\end{array}$ & $\begin{array}{c}0.59 \\
-\end{array}$ & $\begin{array}{l}26.4 \\
24.9\end{array}$ & $\begin{array}{l}293.5 \\
293.2\end{array}$ & $\tilde{0.69}$ & $10 . \overline{40}$ \\
\hline $120-122$ & 150 & $\begin{array}{l}5.61 \\
2.95\end{array}$ & $\begin{array}{c}0.36 \\
-\end{array}$ & $\begin{array}{l}33.0 \\
34.8\end{array}$ & $\begin{array}{l}295.6 \\
297.3\end{array}$ & $\overline{0.53}$ & $8 . \overline{19}$ \\
\hline $130-132$ & 150 & $\begin{array}{l}3.14 \\
1.61\end{array}$ & 0.80 & $\begin{array}{l}38.9 \\
33.6\end{array}$ & $\begin{array}{l}280.0 \\
280.9\end{array}$ & 0.51 & $\overline{2.01}$ \\
\hline $140-142$ & 150 & $\begin{array}{l}0.85 \\
0.09\end{array}$ & $\begin{array}{c}0.39 \\
-\end{array}$ & $\begin{array}{l}67.2 \\
67.4\end{array}$ & $\begin{array}{l}245.8 \\
290.9\end{array}$ & $0 . \overline{11}$ & $\overline{0.23}$ \\
\hline \multicolumn{8}{|l|}{ Section 5} \\
\hline 1-3 & 150 & $\begin{array}{l}1.37 \\
0.26\end{array}$ & $\begin{array}{c}0.52 \\
-\end{array}$ & $\begin{array}{l}72.7 \\
46.7\end{array}$ & $\begin{array}{l}63.3 \\
22.8\end{array}$ & $0 . \overline{19}$ & $0 . \overline{50}$ \\
\hline $11-13$ & 150 & $\begin{array}{l}0.09 \\
0.11\end{array}$ & $\begin{array}{c}0.35 \\
-\end{array}$ & $\begin{array}{r}1.5 \\
-38.3\end{array}$ & $\begin{array}{r}111.2 \\
90.3\end{array}$ & $8 . \overline{18}$ & $\overline{0.31}$ \\
\hline $19-21$ & 150 & $\begin{array}{l}1.50 \\
1.55\end{array}$ & $\begin{array}{c}0.48 \\
-\end{array}$ & $\begin{array}{l}-77.9 \\
-85.1\end{array}$ & $\begin{array}{l}218.6 \\
280.3\end{array}$ & $\overline{1.03}$ & $\overline{3.28}$ \\
\hline $28-30$ & 150 & $\begin{array}{l}2.12 \\
1.99\end{array}$ & $\begin{array}{c}0.58 \\
-\end{array}$ & $\begin{array}{l}-80.4 \\
-77.3\end{array}$ & $\begin{array}{l}277.4 \\
316.8\end{array}$ & $0 . \overline{94}$ & $\overline{3.43}$ \\
\hline $35-37$ & 150 & $\begin{array}{l}1.28 \\
1.10\end{array}$ & 0.26 & $\begin{array}{l}-50.7 \\
-51.1\end{array}$ & $\begin{array}{l}34.2 \\
28.6\end{array}$ & $\overline{0.86}$ & $\overline{4.23}$ \\
\hline $47-49$ & 150 & $\begin{array}{l}2.16 \\
1.84\end{array}$ & 0.28 & $\begin{array}{l}-31.7 \\
-34.6\end{array}$ & $\begin{array}{l}52.2 \\
43.2\end{array}$ & 0.85 & $\overline{6.57}$ \\
\hline $53-55$ & 150 & $\begin{array}{l}2.25 \\
1.97\end{array}$ & $\begin{array}{c}0.28 \\
-\end{array}$ & $\begin{array}{l}-20.8 \\
-24.4\end{array}$ & $\begin{array}{l}53.1 \\
50.1\end{array}$ & $\overline{0.88}$ & $\overline{7.03}$ \\
\hline $61-63$ & 150 & $\begin{array}{l}2.79 \\
2.22\end{array}$ & 0.30 & $\begin{array}{l}-14.2 \\
-19.8\end{array}$ & $\begin{array}{l}55.2 \\
50.5\end{array}$ & $0 . \overline{80}$ & $\overline{7.40}$ \\
\hline $70-72$ & 150 & $\begin{array}{l}3.19 \\
1.97\end{array}$ & 0.46 & $\begin{array}{l}-16.0 \\
-16.3\end{array}$ & $\begin{array}{l}49.1 \\
49.1\end{array}$ & $\overline{0.62}$ & 4.30 \\
\hline
\end{tabular}

Core 46,

\begin{tabular}{|c|c|c|c|c|c|c|c|}
\hline \multicolumn{8}{|l|}{ 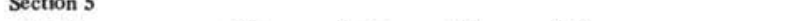 } \\
\hline $77-79$ & 150 & $\begin{array}{l}2.61 \\
2.21\end{array}$ & $\begin{array}{c}0.32 \\
-\end{array}$ & $\begin{array}{l}-15.1 \\
-20.7\end{array}$ & $\begin{array}{l}48.9 \\
44.5\end{array}$ & $0 . \overline{85}$ & $\overline{6.90}$ \\
\hline $108-110$ & 150 & $\begin{array}{l}1.60 \\
1.31\end{array}$ & $\begin{array}{c}0.33 \\
-\end{array}$ & $\begin{array}{l}-15.6 \\
-22.6\end{array}$ & $\begin{array}{l}56.1 \\
51.5\end{array}$ & $\overline{0.82}$ & $3 . \overline{98}$ \\
\hline $118-120$ & 150 & $\begin{array}{l}4.22 \\
3.42\end{array}$ & $\begin{array}{c}0.38 \\
-\end{array}$ & $\begin{array}{l}-21.9 \\
-23.0\end{array}$ & $\begin{array}{l}39.0 \\
36.5\end{array}$ & 0.81 & $8 . \overline{99}$ \\
\hline $129-131$ & 150 & $\begin{array}{l}2.27 \\
2.10\end{array}$ & $\begin{array}{c}0.24 \\
-\end{array}$ & $\begin{array}{l}-21.6 \\
-24.3\end{array}$ & $\begin{array}{l}50.4 \\
42.6\end{array}$ & $0 . \overline{93}$ & $8 . \overline{79}$ \\
\hline $138-140$ & 150 & $\begin{array}{l}2.35 \\
1.86\end{array}$ & $\begin{array}{c}0.29 \\
-\end{array}$ & $\begin{array}{l}-22.7 \\
-28.8\end{array}$ & $\begin{array}{l}50.6 \\
38.9\end{array}$ & $0 . \overline{79}$ & $5 . \overline{10}$ \\
\hline $148-150$ & 150 & $\begin{array}{l}2.70 \\
2.95\end{array}$ & $\begin{array}{c}0.68 \\
-\end{array}$ & $\begin{array}{l}-15.4 \\
-17.0\end{array}$ & $\begin{array}{l}54.9 \\
46.9\end{array}$ & $0 . \overline{92}$ & $3 . \overline{65}$ \\
\hline \multicolumn{8}{|l|}{ Section 6} \\
\hline $4-6$ & 150 & $\begin{array}{l}2.50 \\
2.25\end{array}$ & $\begin{array}{c}0.33 \\
-\end{array}$ & $\begin{array}{l}-15.4 \\
-17.7\end{array}$ & $\begin{array}{l}71.3 \\
67.2\end{array}$ & 0.90 & $\overline{6.82}$ \\
\hline $14-16$ & 150 & $\begin{array}{l}2.10 \\
1.93\end{array}$ & $\begin{array}{c}0.36 \\
-\end{array}$ & $\begin{array}{r}-4.3 \\
-7.8\end{array}$ & $\begin{array}{l}68.6 \\
63.6\end{array}$ & $0 . \overline{92}$ & $5 . \overline{36}$ \\
\hline $21-23$ & 150 & $\begin{array}{l}3.96 \\
5.28\end{array}$ & $\begin{array}{c}0.77 \\
-\end{array}$ & $\begin{array}{r}-12.9 \\
-9.5\end{array}$ & $\begin{array}{l}62.2 \\
41.3\end{array}$ & $1 . \overline{34}$ & $\overline{6.88}$ \\
\hline $28-30$ & 150 & $\begin{array}{r}10.67 \\
9.73\end{array}$ & $\begin{array}{c}1.25 \\
-\end{array}$ & $\begin{array}{l}-2.9 \\
-7.6\end{array}$ & $\begin{array}{l}42.2 \\
41.9\end{array}$ & $0 . \overline{90}$ & $7 . \overline{80}$ \\
\hline $32-34$ & 150 & $\begin{array}{l}5.13 \\
4.70\end{array}$ & $\begin{array}{c}0.32 \\
-\end{array}$ & $\begin{array}{l}-7.0 \\
-9.0\end{array}$ & $\begin{array}{l}52.0 \\
45.4\end{array}$ & $\overline{0.92}$ & $14 . \overline{80}$ \\
\hline $42-44$ & 150 & $\begin{array}{l}2.10 \\
1.79\end{array}$ & 0.25 & $\begin{array}{l}-1.6 \\
-5.5\end{array}$ & $\begin{array}{l}48.8 \\
51.2\end{array}$ & $0 . \overline{85}$ & $\overrightarrow{7.14}$ \\
\hline $50-52$ & 150 & $\begin{array}{l}2.98 \\
2.44\end{array}$ & $\begin{array}{c}0.24 \\
-\end{array}$ & $\begin{array}{l}-13.5 \\
-16.0\end{array}$ & $\begin{array}{l}50.4 \\
53.4\end{array}$ & $\overline{0.82}$ & $10 . \overline{18}$ \\
\hline $60-62$ & 150 & $\begin{array}{l}4.62 \\
3.75\end{array}$ & $\begin{array}{c}0.33 \\
-\end{array}$ & $\begin{array}{l}-30.4 \\
-31.9\end{array}$ & $\begin{array}{l}47.5 \\
45.1\end{array}$ & $0 . \overline{82}$ & $11 . \overline{48}$ \\
\hline $70-72$ & 150 & $\begin{array}{l}2.00 \\
1.73\end{array}$ & $\begin{array}{c}0.40 \\
-\end{array}$ & $\begin{array}{r}-4.8 \\
-10.3\end{array}$ & $\begin{array}{l}53.9 \\
48.4\end{array}$ & $0 . \overline{87}$ & $4 . \overrightarrow{36}$ \\
\hline $80-82$ & 150 & $\begin{array}{l}3.07 \\
2.25\end{array}$ & $\begin{array}{c}0.46 \\
-\end{array}$ & $\begin{array}{l}-25.7 \\
-29.9\end{array}$ & $\begin{array}{l}47.6 \\
62.8\end{array}$ & $0 . \overline{73}$ & $\overline{4.83}$ \\
\hline $90-92$ & 150 & $\begin{array}{l}2.58 \\
1.79\end{array}$ & 0.36 & $\begin{array}{l}-36.1 \\
-39.4\end{array}$ & $\begin{array}{l}56.2 \\
62.7\end{array}$ & $\overline{0.69}$ & $\overline{4.95}$ \\
\hline $98-100$ & 150 & $\begin{array}{l}1.30 \\
0.83\end{array}$ & $\begin{array}{c}0.57 \\
-\end{array}$ & $\begin{array}{r}-8.9 \\
4.2\end{array}$ & $\begin{array}{l}67.4 \\
84.7\end{array}$ & $\overline{0.64}$ & $\overline{1.46}$ \\
\hline $108-110$ & 150 & $\begin{array}{l}2.68 \\
1.87\end{array}$ & $\begin{array}{c}0.66 \\
-\end{array}$ & $\begin{array}{l}-26.2 \\
-35.1\end{array}$ & $\begin{array}{l}48.8 \\
78.6\end{array}$ & $0 . \overline{70}$ & $\overline{2.84}$ \\
\hline
\end{tabular}


TABLE 2 - Continued

\begin{tabular}{|c|c|c|c|c|c|c|c|}
\hline $\begin{array}{l}\text { Core } \\
\text { Interval } \\
(\mathrm{cm})\end{array}$ & $\begin{array}{l}\text { Peak } \\
\text { AF } \\
(\mathrm{oe})\end{array}$ & $\begin{array}{l}J_{S} \\
\left(\times 10^{-5}\right. \\
\text { gauss) }\end{array}$ & $\begin{array}{c}\text { Suscepti- } \\
\text { bility } \\
\text { ( } \times 10^{-5} \\
\text { gauss/oe) }\end{array}$ & $\begin{array}{c}\text { Incli- } \\
\text { nation }\end{array}$ & $\begin{array}{c}\text { Declina- } \\
\text { tion* } \\
\text { (relative } \\
\text { degrees) }\end{array}$ & $\begin{array}{l}\text { Rela- } \\
\text { tive } \\
\text { Inten- } \\
\text { sity }\end{array}$ & $\begin{array}{l}\text { Ratio of } \\
\text { NRM Afte } \\
\text { AFD to } \\
\text { Initial Sus } \\
\text { ceptibility }\end{array}$ \\
\hline \multicolumn{8}{|l|}{$\begin{array}{l}\text { Core 47, } \\
\text { Section } 1\end{array}$} \\
\hline $3-5$ & 150 & $\begin{array}{l}1.04 \\
0.66\end{array}$ & $\begin{array}{c}0.30 \\
-\end{array}$ & $\begin{array}{l}-19.3 \\
-29.2\end{array}$ & $\begin{array}{l}72.5 \\
61.9\end{array}$ & $\overline{0.63}$ & 2.19 \\
\hline $10-12$ & 150 & $\begin{array}{l}0.81 \\
0.64\end{array}$ & 0.30 & $\begin{array}{l}-21.4 \\
-28.0\end{array}$ & $\begin{array}{l}49.8 \\
43.6\end{array}$ & $0 . \overline{79}$ & $2 . \overline{14}$ \\
\hline $20-22$ & 150 & $\begin{array}{l}0.93 \\
1.00\end{array}$ & 0.30 & $\begin{array}{l}-11.7 \\
-23.1\end{array}$ & $\begin{array}{l}41.4 \\
22.5\end{array}$ & $1 . \overline{07}$ & 3.31 \\
\hline $30-32$ & 150 & $\begin{array}{l}0.78 \\
0.61\end{array}$ & $\begin{array}{c}0.31 \\
-\end{array}$ & $\begin{array}{r}-6.3 \\
-26.9\end{array}$ & $\begin{array}{l}44.3 \\
58.5\end{array}$ & $\overline{0.78}$ & $1 . \overline{95}$ \\
\hline $49-42$ & 150 & $\begin{array}{l}1.35 \\
1.18\end{array}$ & $\stackrel{0.31}{-}$ & $\begin{array}{l}-5.1 \\
-9.2\end{array}$ & $\begin{array}{l}44.6 \\
27.2\end{array}$ & $\overline{0.87}$ & 3.78 \\
\hline $50-52$ & 150 & $\begin{array}{l}0.89 \\
1.01\end{array}$ & $\begin{array}{c}0.39 \\
-\end{array}$ & $\begin{array}{l}-27.2 \\
-34.2\end{array}$ & $\begin{array}{l}71.0 \\
40.5\end{array}$ & 1.13 & 2.59 \\
\hline $58-60$ & 150 & $\begin{array}{l}0.69 \\
0.91\end{array}$ & $\begin{array}{c}0.44 \\
-\end{array}$ & $\begin{array}{r}0.4 \\
-12.0\end{array}$ & $\begin{array}{l}65.1 \\
21.7\end{array}$ & 1.31 & 2.04 \\
\hline $64-66$ & 150 & $\begin{array}{l}3.78 \\
2.86\end{array}$ & $\begin{array}{c}0.26 \\
-\end{array}$ & $\begin{array}{l}-18.7 \\
-22.2\end{array}$ & $\begin{array}{l}131.3 \\
127.4\end{array}$ & $\overline{0.76}$ & $11 . \overline{05}$ \\
\hline $73-75$ & 150 & $\begin{array}{l}2.82 \\
2.58\end{array}$ & 0.53 & $\begin{array}{l}-19.1 \\
-19.1\end{array}$ & $\begin{array}{l}143.4 \\
141.4\end{array}$ & $\overrightarrow{0.90}$ & 4.87 \\
\hline $84-86$ & 150 & $\begin{array}{l}2.35 \\
1.90\end{array}$ & 0.28 & $\begin{array}{l}-23.3 \\
-19.1\end{array}$ & $\begin{array}{l}128.1 \\
135.4\end{array}$ & $\overline{0.81}$ & $\overline{6.80}$ \\
\hline $94-96$ & 150 & $\begin{array}{l}2.95 \\
1.92\end{array}$ & $\frac{0.38}{-}$ & $\begin{array}{l}-16.3 \\
-10.4\end{array}$ & $\begin{array}{l}132.1 \\
120.1\end{array}$ & $\overline{0.65}$ & 5.04 \\
\hline $104-106$ & 150 & $\begin{array}{l}3.62 \\
2.46\end{array}$ & $\stackrel{0.30}{-}$ & $\begin{array}{l}-21.4 \\
-14.4\end{array}$ & $\begin{array}{l}136.2 \\
131.6\end{array}$ & $\overline{0.68}$ & $8 . \overline{21}$ \\
\hline $114-116$ & 150 & $\begin{array}{l}1.90 \\
1.16\end{array}$ & $\underset{-}{0.31}$ & $\begin{array}{r}-3.4 \\
-19.6\end{array}$ & $\begin{array}{l}143.7 \\
132.1\end{array}$ & $\overline{0.61}$ & 3.74 \\
\hline $124-126$ & 150 & $\begin{array}{l}2.36 \\
1.62\end{array}$ & $\begin{array}{c}0.34 \\
-\end{array}$ & $\begin{array}{l}-11.3 \\
-13.8\end{array}$ & $\begin{array}{l}141.7 \\
126.7\end{array}$ & $\overline{0.69}$ & $\overline{4.79}$ \\
\hline $134-136$ & 150 & $\begin{array}{l}2.33 \\
2.09\end{array}$ & $\underset{-}{0.32}$ & $\begin{array}{l}-21.6 \\
-29.2\end{array}$ & $\begin{array}{l}148.7 \\
146.2\end{array}$ & $\tilde{0.90}$ & $\overline{6.55}$ \\
\hline $144-146$ & 150 & $\begin{array}{l}2.50 \\
1.38\end{array}$ & 0.31 & $\begin{array}{l}-21.1 \\
-14.3\end{array}$ & $\begin{array}{l}114.1 \\
121.0\end{array}$ & 0.55 & $\overline{4.43}$ \\
\hline
\end{tabular}

Section 2

\begin{tabular}{|c|c|c|c|c|c|c|c|}
\hline $1-3$ & 150 & $\begin{array}{l}3.50 \\
2.40\end{array}$ & $\begin{array}{c}0.28 \\
-\end{array}$ & $\begin{array}{l}-22.0 \\
-24.7\end{array}$ & $\begin{array}{l}22.9 \\
19.4\end{array}$ & $\overline{0.69}$ & $8 . \overline{63}$ \\
\hline $12-14$ & 150 & $\begin{array}{l}2.06 \\
1.42\end{array}$ & $\begin{array}{c}0.26 \\
-\end{array}$ & $\begin{array}{l}-23.5 \\
-26.1\end{array}$ & $\begin{array}{l}29.5 \\
25.2\end{array}$ & $\overline{0.69}$ & $5 . \overline{46}$ \\
\hline $22-24$ & 150 & $\begin{array}{l}1.60 \\
1.15\end{array}$ & $\begin{array}{c}0.26 \\
-\end{array}$ & $\begin{array}{r}-14.5 \\
-8.1\end{array}$ & $\begin{array}{l}33.4 \\
36.0\end{array}$ & $0 . \overline{72}$ & $\overline{4.43}$ \\
\hline $32-34$ & 150 & $\begin{array}{l}1.52 \\
0.99\end{array}$ & $\begin{array}{c}0.28 \\
-\end{array}$ & $\begin{array}{r}-8.5 \\
-11.1\end{array}$ & $\begin{array}{l}37.7 \\
20.1\end{array}$ & $0 . \overline{65}$ & 3.53 \\
\hline $52-54$ & 150 & $\begin{array}{l}2.08 \\
1.36\end{array}$ & $\begin{array}{c}0.28 \\
-\end{array}$ & $\begin{array}{r}-10.9 \\
-4.9\end{array}$ & $\begin{array}{l}28.7 \\
26.3\end{array}$ & $0 . \overline{65}$ & $4 . \overline{82}$ \\
\hline $62-64$ & 150 & $\begin{array}{l}4.41 \\
2.72\end{array}$ & $\begin{array}{c}0.42 \\
-\end{array}$ & $\begin{array}{l}-23.5 \\
-24.6\end{array}$ & $\begin{array}{r}24.3 \\
7.6\end{array}$ & $\overline{0.61}$ & $6 . \overline{22}$ \\
\hline $73-75$ & 150 & $\begin{array}{l}5.46 \\
3.76\end{array}$ & $\begin{array}{c}0.81 \\
-\end{array}$ & $\begin{array}{l}-18.2 \\
-21.5\end{array}$ & $\begin{array}{l}25.8 \\
22.4\end{array}$ & $\overline{0.69}$ & $4 . \overline{64}$ \\
\hline $77-79$ & 150 & $\begin{array}{l}3.86 \\
3.12\end{array}$ & $\begin{array}{c}0.27 \\
-\end{array}$ & $\begin{array}{l}-24.9 \\
-27.8\end{array}$ & $\begin{array}{l}19.5 \\
17.3\end{array}$ & $\overline{0.81}$ & $11 . \overline{90}$ \\
\hline $86-88$ & 150 & $\begin{array}{l}3.95 \\
3.19\end{array}$ & $\begin{array}{c}0.29 \\
-\end{array}$ & $\begin{array}{l}-27.6 \\
-28.4\end{array}$ & $\begin{array}{l}22.7 \\
19.1\end{array}$ & $\tilde{0.81}$ & $11 \overline{0} \overline{0}$ \\
\hline $98-100$ & 150 & $\begin{array}{l}2.00 \\
1.74\end{array}$ & $\begin{array}{c}0.29 \\
-\end{array}$ & $\begin{array}{r}-8.5 \\
-12.8\end{array}$ & $\begin{array}{l}21.5 \\
14.5\end{array}$ & $\overline{0.87}$ & $\overline{6.00}$ \\
\hline $107-109$ & 150 & $\begin{array}{l}1.07 \\
0.92\end{array}$ & $\begin{array}{c}0.28 \\
-\end{array}$ & $\begin{array}{l}42.5 \\
26.9\end{array}$ & $\begin{array}{l}37.8 \\
24.1\end{array}$ & $\overline{0.86}$ & $3 . \overline{20}$ \\
\hline $114-116$ & 150 & $\begin{array}{l}0.76 \\
0.85\end{array}$ & $\begin{array}{c}0.24 \\
-\end{array}$ & $\begin{array}{l}49.2 \\
13.7\end{array}$ & $\begin{array}{r}11.2 \\
1.8\end{array}$ & $1 . \overline{12}$ & $3 . \overline{80}$ \\
\hline
\end{tabular}

Core 47,
Section 2

\begin{tabular}{cccccccc}
$128-130$ & & 1.55 & 0.48 & 9.0 & 36.3 & - & - \\
& 150 & 1.49 & - & -4.7 & 12.0 & 0.96 & 3.10 \\
$138-140$ & & 4.06 & 0.42 & -12.4 & 40.3 & - & - \\
& 150 & 3.02 & - & -16.9 & 37.8 & 0.74 & 7.20 \\
$146-148$ & & 2.98 & 0.23 & -16.3 & 37.2 & - & - \\
& 150 & 2.20 & - & -17.4 & 33.9 & 0.74 & 9.60 \\
Section 3 & & & & & & & \\
$2-4$ & \multirow{2}{*}{150} & 3.17 & 0.21 & -26.1 & 48.0 & - & - \\
$7-9$ & & 3.59 & - & -26.5 & 43.9 & 0.82 & 12.30 \\
\hline
\end{tabular}

TABLE 2 - Continued

\begin{tabular}{|c|c|c|c|c|c|c|c|}
\hline $\begin{array}{l}\text { Core } \\
\text { Interval } \\
(\mathrm{cm})\end{array}$ & $\begin{array}{l}\text { Peak } \\
\text { AF } \\
\text { (oe) }\end{array}$ & $\begin{array}{c}J_{S} \\
\text { (× } 10^{-5} \\
\text { gauss) }\end{array}$ & $\begin{array}{l}\text { Suscepti- } \\
\text { bility } \\
\text { ( } \times 10^{-5} \\
\text { gauss/oe })\end{array}$ & $\begin{array}{l}\text { Incli- } \\
\text { nation }\end{array}$ & $\begin{array}{l}\text { Declina- } \\
\text { tion* } \\
\text { (relative } \\
\text { degrees) }\end{array}$ & $\begin{array}{l}\text { Rela- } \\
\text { tive } \\
\text { Inten- } \\
\text { sity }\end{array}$ & $\begin{array}{l}\text { Ratio of } \\
\text { NRM After } \\
\text { AFD to } \\
\text { Initial Sus- } \\
\text { ceptibility }\end{array}$ \\
\hline & 150 & 2.30 & - & -23.3 & 42.6 & 0.75 & 4.42 \\
\hline $19-21$ & 150 & $\begin{array}{l}1.72 \\
1.57\end{array}$ & $\begin{array}{c}0.59 \\
-\end{array}$ & $\begin{array}{l}-14.8 \\
-16.6\end{array}$ & $\begin{array}{l}48.0 \\
38.9\end{array}$ & $\overline{0.91}$ & $\overline{2.66}$ \\
\hline $29-31$ & 150 & $\begin{array}{l}3.31 \\
2.28\end{array}$ & 0.24 & $\begin{array}{l}-22.5 \\
-27.8\end{array}$ & $\begin{array}{l}195.7 \\
195.1\end{array}$ & $\overline{0.69}$ & $9 . \overline{50}$ \\
\hline $38-40$ & 150 & $\begin{array}{l}2.65 \\
2.33\end{array}$ & $\begin{array}{c}0.56 \\
-\end{array}$ & $\begin{array}{r}-4.0 \\
-12.0\end{array}$ & $\begin{array}{l}212.7 \\
202.7\end{array}$ & $\overline{0.88}$ & $4 . \overline{16}$ \\
\hline $55-57$ & 150 & $\begin{array}{l}0.45 \\
0.81\end{array}$ & $\begin{array}{c}0.39 \\
-\end{array}$ & $\begin{array}{l}42.0 \\
22.1\end{array}$ & $\begin{array}{l}185.3 \\
172.5\end{array}$ & $1 . \overline{80}$ & $2 . \overline{28}$ \\
\hline $61-63$ & 150 & $\begin{array}{l}0.99 \\
1.00\end{array}$ & $\begin{array}{c}0.28 \\
-\end{array}$ & $\begin{array}{l}16.7 \\
-5.9\end{array}$ & $\begin{array}{l}172.9 \\
158.8\end{array}$ & $1 . \overline{01}$ & 3.57 \\
\hline $73-75$ & 150 & $\begin{array}{l}2.40 \\
1.84\end{array}$ & $\begin{array}{c}0.47 \\
-\end{array}$ & $\begin{array}{l}34.8 \\
28.5\end{array}$ & $\begin{array}{l}114.4 \\
116.2\end{array}$ & $0 . \overline{77}$ & $3 . \overline{91}$ \\
\hline $88-90$ & 150 & $\begin{array}{l}3.73 \\
3.26\end{array}$ & $\begin{array}{c}0.44 \\
-\end{array}$ & $\begin{array}{l}47.7 \\
29.3\end{array}$ & $\begin{array}{l}1.4 \\
1.3\end{array}$ & $\overline{0.87}$ & $\overline{7.36}$ \\
\hline $98-100$ & 150 & $\begin{array}{l}6.25 \\
3.74\end{array}$ & $\begin{array}{c}0.39 \\
-\end{array}$ & $\begin{array}{l}25.0 \\
27.3\end{array}$ & $\begin{array}{l}3.8 \\
8.1\end{array}$ & $\overline{0.59}$ & $9 . \overline{46}$ \\
\hline $108-110$ & 150 & $\begin{array}{l}4.78 \\
2.94\end{array}$ & $\begin{array}{c}0.25 \\
-\end{array}$ & $\begin{array}{l}25.8 \\
24.6\end{array}$ & $\begin{array}{l}15.4 \\
19.7\end{array}$ & $\overline{0.62}$ & $11 . \overline{85}$ \\
\hline $118-120$ & 150 & $\begin{array}{l}4.92 \\
3.01\end{array}$ & $\begin{array}{c}0.26 \\
-\end{array}$ & $\begin{array}{l}29.1 \\
21.3\end{array}$ & $\begin{array}{l}12.1 \\
18.3\end{array}$ & $\overline{0.61}$ & $11 . \overline{54}$ \\
\hline $128-130$ & 150 & $\begin{array}{l}5.03 \\
2.97\end{array}$ & $\begin{array}{c}0.29 \\
-\end{array}$ & $\begin{array}{l}30.9 \\
25.0\end{array}$ & $\begin{array}{l}7.6 \\
4.6\end{array}$ & $\overline{0.59}$ & $10 . \overline{23}$ \\
\hline $138-140$ & 150 & $\begin{array}{l}4.59 \\
3.22\end{array}$ & $\begin{array}{c}0.42 \\
-\end{array}$ & $\begin{array}{l}30.3 \\
36.4\end{array}$ & $\begin{array}{r}3.7 \\
-9.1\end{array}$ & $\overline{0.70}$ & $\overline{7.65}$ \\
\hline $148-150$ & 150 & $\begin{array}{l}5.60 \\
3.11\end{array}$ & $\begin{array}{c}0.36 \\
-\end{array}$ & $\begin{array}{l}32.6 \\
26.4\end{array}$ & $\begin{array}{l}14.0 \\
12.0\end{array}$ & $0 . \overline{56}$ & $8 . \overline{71}$ \\
\hline \multicolumn{8}{|l|}{ Section 4} \\
\hline $8-10$ & 150 & $\begin{array}{l}5.73 \\
3.65\end{array}$ & $\begin{array}{c}0.28 \\
-\end{array}$ & $\begin{array}{l}28.4 \\
28.6\end{array}$ & $\begin{array}{l}180.8 \\
182.3\end{array}$ & $\overline{0.64}$ & $13 . \overline{09}$ \\
\hline $18-20$ & 150 & $\begin{array}{l}3.45 \\
2.04\end{array}$ & $\begin{array}{c}0.28 \\
-\end{array}$ & $\begin{array}{l}28.8 \\
18.5\end{array}$ & $\begin{array}{l}186.6 \\
199.1\end{array}$ & $0 . \overline{59}$ & $\overline{7.26}$ \\
\hline $28-30$ & 150 & $\begin{array}{l}4.00 \\
2.68\end{array}$ & $\begin{array}{c}0.29 \\
-\end{array}$ & $\begin{array}{l}31.8 \\
26.8\end{array}$ & $\begin{array}{l}197.2 \\
217.2\end{array}$ & $\overline{0.67}$ & $9 . \overline{24}$ \\
\hline $38-40$ & 150 & $\begin{array}{l}3.82 \\
2.42\end{array}$ & $\begin{array}{c}0.24 \\
-\end{array}$ & $\begin{array}{l}22.8 \\
24.4\end{array}$ & $\begin{array}{l}187.3 \\
198.3\end{array}$ & $\overline{0.63}$ & $10 . \overline{03}$ \\
\hline $58-60$ & 150 & $\begin{array}{l}2.75 \\
1.65\end{array}$ & $\begin{array}{c}0.34 \\
-\end{array}$ & $\begin{array}{l}24.0 \\
24.5\end{array}$ & $\begin{array}{l}183.1 \\
176.4\end{array}$ & $\overline{0.60}$ & $\overline{4.85}$ \\
\hline $68-70$ & 150 & $\begin{array}{l}5.45 \\
3.92\end{array}$ & $\begin{array}{c}0.39 \\
-\end{array}$ & $\begin{array}{l}21.1 \\
21.5\end{array}$ & $\begin{array}{l}280.3 \\
280.6\end{array}$ & $0 . \overline{71}$ & $\overline{9.92}$ \\
\hline $73-75$ & 150 & $\begin{array}{l}3.96 \\
2.97\end{array}$ & $\begin{array}{c}0.47 \\
-\end{array}$ & $\begin{array}{l}29.4 \\
28.4\end{array}$ & $\begin{array}{l}180.3 \\
183.7\end{array}$ & $\overline{0.80}$ & $\overline{6.32}$ \\
\hline
\end{tabular}

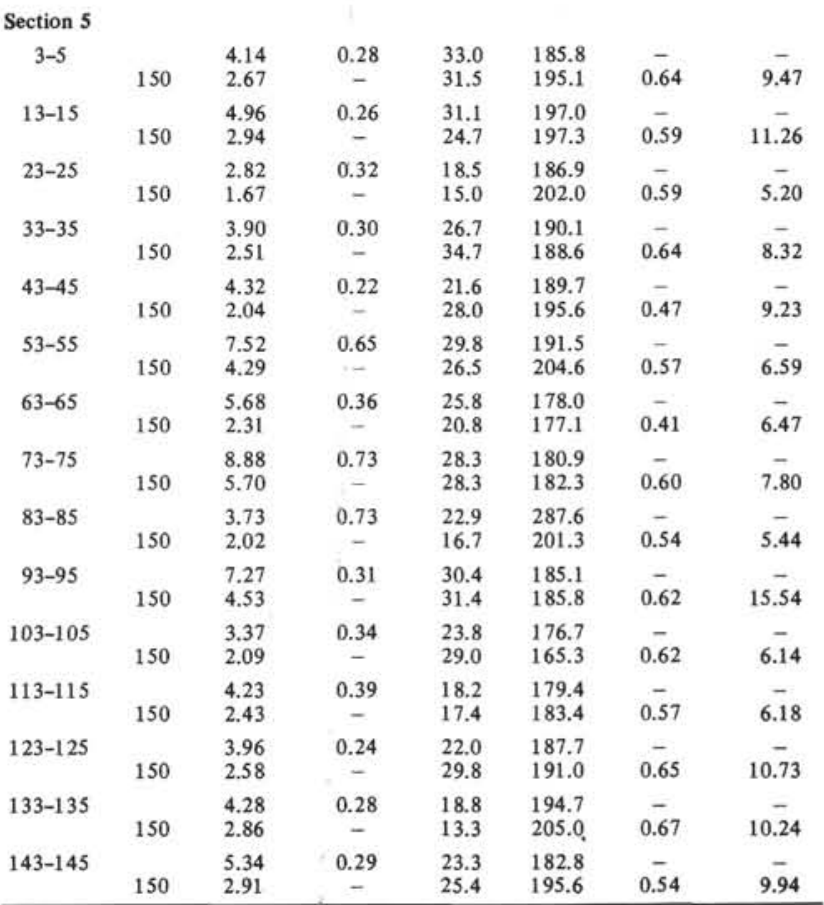


TABLE 2 - Continued

\begin{tabular}{|c|c|c|c|c|c|c|c|}
\hline $\begin{array}{l}\text { Core } \\
\text { Interval } \\
(\mathrm{cm})\end{array}$ & $\begin{array}{l}\text { Peak } \\
\text { AF } \\
\text { (oe) }\end{array}$ & $\begin{array}{c}J_{S} \\
\left(\times 10^{-5}\right. \\
\text { gauss })\end{array}$ & $\begin{array}{c}\text { Suscepti- } \\
\text { bility } \\
\left(\times 10^{-5}\right. \\
\text { gauss/oe })\end{array}$ & $\begin{array}{l}\text { Incli- } \\
\text { nation }\end{array}$ & $\begin{array}{c}\text { Declina- } \\
\text { tion** } \\
\text { (relative } \\
\text { degrees) }\end{array}$ & $\begin{array}{l}\text { Rela- } \\
\text { tive } \\
\text { Inten- } \\
\text { sity }\end{array}$ & $\begin{array}{l}\text { Ratio of } \\
\text { NRM After } \\
\text { AFD to } \\
\text { Initial Sus- } \\
\text { ceptibility }\end{array}$ \\
\hline \multicolumn{8}{|c|}{ Section 6} \\
\hline $3-5$ & 150 & $\begin{array}{l}1.85 \\
1.66\end{array}$ & $\begin{array}{c}0.30 \\
-\end{array}$ & $\begin{array}{l}36.2 \\
18.0\end{array}$ & $\begin{array}{l}281.3 \\
252.3\end{array}$ & $\overline{0.90}$ & $5 . \overline{54}$ \\
\hline $23-25$ & 150 & $\begin{array}{l}3.35 \\
2.07\end{array}$ & 0.25 & $\begin{array}{l}16.1 \\
16.4\end{array}$ & $\begin{array}{l}276.5 \\
277.8\end{array}$ & $0 . \overline{61}$ & $8 . \overline{17}$ \\
\hline $33-35$ & 150 & $\begin{array}{l}3.98 \\
2.34\end{array}$ & $\begin{array}{c}0.21 \\
-\end{array}$ & $\begin{array}{l}23.5 \\
14.0\end{array}$ & $\begin{array}{l}272.3 \\
274.6\end{array}$ & 0.59 & $11 . \overline{20}$ \\
\hline $43-45$ & 150 & $\begin{array}{l}3.72 \\
2.44\end{array}$ & $\begin{array}{c}0.20 \\
-\end{array}$ & $\begin{array}{l}23.5 \\
30.1\end{array}$ & $\begin{array}{l}272.8 \\
273.4\end{array}$ & $\overline{0.65}$ & $12 . \overline{09}$ \\
\hline $53-55$ & 150 & $\begin{array}{l}6.51 \\
3.76\end{array}$ & $\underset{-}{0.31}$ & $\begin{array}{l}27.3 \\
25.4\end{array}$ & $\begin{array}{l}275.1 \\
278.5\end{array}$ & $\overline{0.58}$ & $12 . \overline{18}$ \\
\hline $67-69$ & 150 & $\begin{array}{l}4.43 \\
3.33\end{array}$ & $\begin{array}{c}0.28 \\
-\end{array}$ & $\begin{array}{l}15.8 \\
10.0\end{array}$ & $\begin{array}{l}277.0 \\
277.4\end{array}$ & $\overline{0.75}$ & $11 \overline{85}$ \\
\hline $87-89$ & 150 & $\begin{array}{l}4.34 \\
2.76\end{array}$ & $\begin{array}{c}0.31 \\
-\end{array}$ & $\begin{array}{r}11.1 \\
6.0\end{array}$ & $\begin{array}{l}270.6 \\
281.9\end{array}$ & $\overline{0.64}$ & $8 . \overline{96}$ \\
\hline
\end{tabular}

Ito, H., 1963. Paleomagnetic study on Kyushu outer zone granite. Annual Prog. Rept. Rock Magnetism Res. Group Japan, 87-90.

1965. Paleomagnetic study on a granitic rock mass with normal and reverse natural remanent magnetization. J. Geomagnet. Geoelec., 17, 113-120.

1970. Polarity transitions of the geomagnetic field deduced from the natural remanent magnetization of Tertiary and Quaternary rocks in southwest Japan. J. Geomagnet. Geoelec., 22, 273-291.

Kawai, N., Nakajima, T., Yaskawa, K., Hirooka, K., and Kobayashi, K., 1973. The oscillation of field in the Matuyama geomagnetic epoch. Proc. Japan Acad., 49, 619-622.

Kawai, N., Nakajima, T., Hirooka, K., and Kobayashi, K., 1973. The transition of field at the Brunhes and Jaramillo boundaries in the Matuyama geomagnetic epoch. Proc. Japan Acad., 49, 820-824.

Kawai, N., Otofuji, Y., and Kobayashi, K., 1976. Paleomagnetic study of deep-sea sediments using thin sections. $J$. Geomagnet. Geoelec., 28, 395-412.
Kawai, N., Sato, T., Sueishi, T., and Kobayashi, K., 1977. Paleomagnetic study of deep-sea sediments from the Meranesia Basin. J. Geomagnet. Geoelec., 29, 211-223.

Larson, E. E., Watson, D. E., and Jennings, W., 1971. Regional comparison of a Miocene geomagnetic transition in Oregon and Nevada. Earth Planet. Sci. Lett., 11, 391-400.

Momose, K., 1963. Studies on the variations of the Earth's magnetic field during Pliocene times. Bull. Earthquake Res. Inst. Tokyo Univ., 41, 487-534.

Nomura, S., 1967. On the properties of the paleomagnetic polarity of the Neogene and Quaternary volcanic rocks in Japan. J. Liberal Art Sci. Gumma Univ. Jap., 1, 11-35.

Opdyke, N. D., Kent, D. V., and Lowrie, W., 1973. Details of magnetic polarity transitions recorded in a high deposition rate deep-sea core. Earth Planet. Sci. Lett., 20, 315-324.

Readman, P. W., and O'Reilly, W., 1972. Magnetic properties of oxidized cation-deficient titanomagnetite $(\mathrm{Fe}, \mathrm{Ti}, \square)_{3} \mathrm{O}_{4}$. J. Geomagnet. Geoelec., 24, 69-90.

Vlasov, A. Y., and Kovalenko, G. V., 1963. Magnetism of transitional layers between normal and reversed remanent magnetizations. Izv. Akad. Nauk, Geophys. Ser., No. 4, 552-560.

Watkins, N. D., 1963. Behaviour of the geomagnetic field during the Miocene period in south-eastern Oregon. Nature, $197,126-128$.

Wilson, R. L., Dagley, P., and McCormack, A. G., 1972. Paleomagnetic evidence about the source of the geomagnetic field. Geophys. J. Royal Astron. Soc., 28, 213-224.

Yaskawa, K., Nakajima, T., Kawai, N., Torii, M., Natsuhara, N., and Horie, S., 1973. Paleomagnetism of a core from Lake Biwa (1). J. Geomagnet. Geoelec., 25, 447-474.

Yukutake, T., 1968. Free decay of non-dipole components of the geomagnetic field. Phys. Earth Planet. Interiors, 1, 93-96.

van Zijl, J. S. V., Graham, K. W. T., and Hales, A. L., 1962a. The paleomagnetism of the Stormsberg lavas of South Africa. Geophys. J. Royal Astron. Soc., 7, 23-39.

1962b. The paleomagnetism of the Stormsberg lavas (II). Geophys. J. Royal Astron. Soc., 7, 169-181. 